\title{
Visualising Equivalent System Networks in the NetLP optimisation of water distribution systems
}

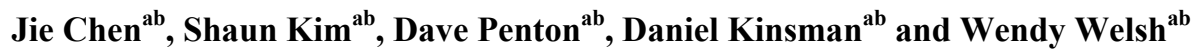 \\ ${ }^{a}$ CSIRO Water for a Healthy Country National Research Flagship, CSIRO Land and Water, GPO Box 1666, \\ Canberra, ACT 2601, Australia \\ ${ }^{b}$ eWater Cooperative Research Centre, University of Canberra, ACT 2601, Australia \\ Email: jie.chen@csiro.au
}

\begin{abstract}
System simulation modeling plays an important role in water resources management in Australia and other countries. Network linear programming (NetLP) solver-based modelling software e.g. REALM, WathNet, MODSIM and Source Integrated Modelling System (Source IMS) enable water distribution modelling of river systems with multiple supply paths using an optimisation engine in contrary to rules-based ordering system. A NetLP solver-based ordering system usually needs to translate the network elements in a river system, e.g. inflows, storages, confluences and regulated splitters (a splitter represents a branch in the river system, such as an anabranch; and a regulated splitter represents a branch where the flow down at least one branch can be varied by use of a structure), into an Equivalent System Network (ESN). The translated ESN incorporates the arc capacities and costs defined by the system or user to reflect the linear constraints and preferences for water distribution. NetLP solvers are called iteratively to achieve specified convergence criteria for nonlinear or piece-wise linear relationships within the model of the water supply system. Existing software such as REALM or the early internal release versions of Source IMS have no capability to visualise the generated ESNs, making it less transparent for users to understand the solutions of NetLP solvers by reading through a text file recording the nodes and arcs of the network.
\end{abstract}

In this paper, we propose a visualisation aid for interpreting the ESNs in Source IMS based on a third-party tool, Graphviz. Firstly, we introduce the implementation of exporting ESNs to the opensource Graphviz, which provides the features of subgraphs and clusters in the DOT language, as shown in Figure 2, an example of a simple river system. Then the usefulness of the visualisations is illustrated with two case studies using NetLP functionality in Source IMS. The first case of visualisation shows that translation of a regulated splitter into an ESN has allowed the NetLP to make an arbitrary choice of supply path. In fact, the modeler would prefer to favour the river channel rather than the regulated effluent to convey excess water. With this knowledge, adding a minor cost difference to the effluent arc causes Source IMS to release water down the path preferred by the modeler (left branch of the splitter shown in Figure 2 and 4). The second case deals with the cost ambiguities in a complicated river system network, which leads to different solutions using different NetLP solvers. The visualisation shows multiple paths from different storages to the extraction point with exactly the same

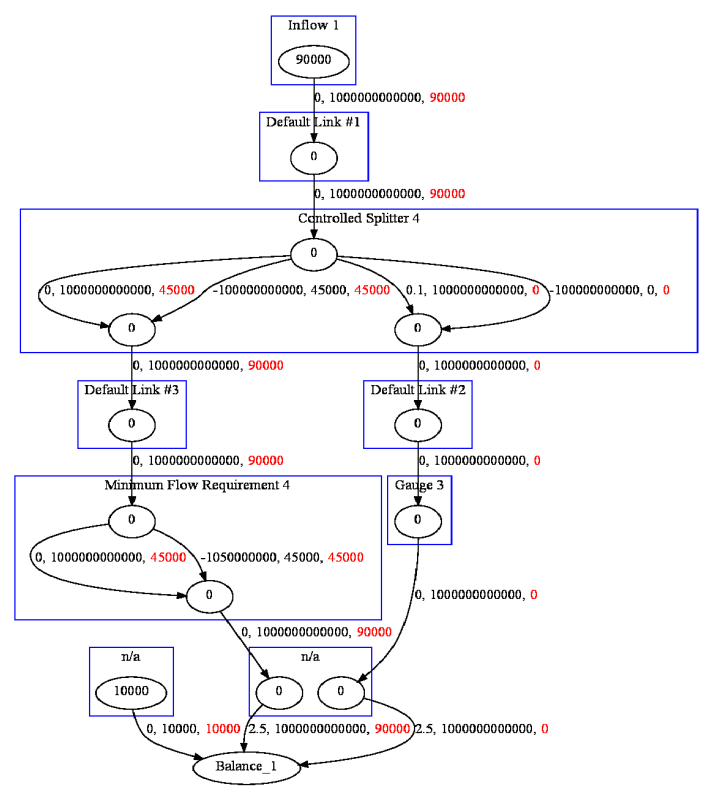

Figure 2. Example of visualisation of an ESN. Each arc is labeled with three numbers in order representing cost, capacity $\left(\mathrm{m}^{3}\right)$, and computed total cost. In this case, the Source IMS developers created an automated solution to handle such cost ambiguities that works effectively in tested river system networks.

Keywords: Information Visualization, Modelling Tools, River Systems, Network Linear Programming, Source IMS 
Chen et al., Visualising Equivalent System Networks in the NetLP Optimisation of Water Distribution Systems

\section{INTRODUCTION}

System simulation modeling plays an important role in water resources management in Australia and other countries. Specifically, water managers are interested in more than obtaining a qualitative understanding of the water distributions in river systems. They must be able to deal quantitatively with interrelations between factors and to predict and optimise the distribution of water resources available in the systems with the help of mathematical solvers. This is due to the complexity of hydrologic phenomena and the requirements of water resource management, which makes it difficult to apply rigorous deductive reasoning (Linsley et al., 1982, p.5), e.g. rules-based ordering system is difficult to build to meet demands with multiple supply paths. Therefore Network Linear Program (NetLP) solver-based modelling software e.g. REALM (REALM, 2011; Perera et al., 2005), WathNet (Kuczera, 1992), MODSIM (Labadie 2006, MODSIM 2011) and Source Integrated Modelling System (Source IMS) (Delgado et al., 2011; Penton and Gilmore, 2009). have been designed to enable analysis of optimised water distribution in river systems with multiple supply paths. Labadie (2004) surveys a range of other solutions to the water distribution problem including genetic algorithms; however, the trade-off of performance tends to favour simpler approaches. Source IMS (Welsh et al 2011), which we will focus on, has two modes for determining how regulated structures such as storages should operate to distribute water to downstream demands. The two modes are rules-based, and an iterative solution to a network linear program. This paper focuses on the second mode.

In Source IMS, modellers build a river systems model containing river systems network elements: inflows, storages, confluences, splitters (a splitter represents a branch in the river system, such as an anabranch; and a regulated/controlled splitter represents a branch where the flow down at least one branch can be varied by use of a structure), loss nodes and so on. The simulation engine translates these river systems network elements into an Equivalent System Network (ESN) (Perera et al., 2005) that an optimiser can solve. As with (Perera et al., 2005), the ESN for Source IMS is a standard form of network linear program with arcs and nodes (See Bertsekas 1998). The translated ESN incorporates the capacities and costs defined by the system or user to reflect the linear constraints and preferences for water distribution, as an input to NetLP solvers. Note that the costs are relative penalties, not in real dollars, to attempt to satisfy water assignment criteria during each simulation time step. NetLP solvers are called iteratively to solve the input ESN to achieve a certain convergence criteria with the consideration of nonlinearity or piece-wise linear relationship of discharge or loss. There are different ways of building ESNs and updating them from iteration to iteration, e.g. updating the capacity of the loss arc of a loss node according to the last iteration's inflow. Therefore, there is often a difference between the NetLP solver's solution and what a modeller might expect. In some circumstances, Ilich $(2008,2009)$ describes problems where this approach produces non-optimal solutions to the water distribution problem. A problem dealt with in this paper is that ambiguities in costs of different flow paths can lead to different solutions using different NetLP solvers.

Existing software such as REALM or the early internal release versions of Source IMS have no capability to visualise the generated ESNs, so it is very difficult for users to understand the solutions of NetLP solvers by reading through the text file that describes the nodes and arcs of the network. In this paper, visualisation of ESNs is advocated throughout the lifecycle of optimisation-based modelling practices, because it can support the building and understanding the optimisation models, adding transparency with appropriate representations of the models (Jones, 1996).

For this reason, Source IMS has introduced a visualisation capability, based on the third-party tool Graphviz (2011), using the DOT language to provide the user with a rapid means of viewing the cost-capacity structure of the ESN network behind their model. This aims to provide the modeller with an improved understanding of how the network linear optimiser is behaving in response to the networks they have constructed. The usefulness of the visualisations is illustrated by two case studies using NetLP functionality in Source IMS. The first case of visualisation shows that the translation of the regulated splitter (or controlled splitter in Source IMS) into an ESN has allowed the NetLP to make an arbitrary choice of supply path. In fact, the modeler would prefer to favour the main channel to release excess water. The second case involves cost ambiguities in a complicated river system network with multiple storages with multiple flow paths. The cost ambiguities with multiple paths from different storages are studied with the aid of visualizations of ESNs. 
Chen et al., Visualising Equivalent System Networks in the NetLP Optimisation of Water Distribution Systems

\section{METHODS}

\subsection{Choice of Visualisation Package}

Many visualisation packages present diagrams or schematics and could be used to visualize ESNs. The choice to export to Graphviz (Gansner and North, 1999) was chosen because it is satisfied the following criteria:

- It must have a simple and well-described input file format, preferably text-based.

- It must be easy to use.

- It must be readily available.

- It must be affordable and distributable.

In addition to meeting these requirements, Graphviz has high-quality static and dynamic layout algorithms, and the ability to handle sizable graphs, including a complement of file-stream graph processors. The Graphviz layout programs take descriptions of graphs in a simple text language, and make diagrams in useful formats, such as images and SVG for web pages, PDF or Postscript for inclusion in other documents, or display in an interactive graph browser. Graphviz has many useful features for concrete diagrams, such as options for colours, fonts, tabular node layouts, line styles, hyperlinks, and rolland custom shapes. It has important applications in networking, bioinformatics, software engineering, database and web design, machine learning, and in visual interfaces for other technical domains. Graphviz supports the DOT language that draws directed graphs as hierarchies.

\subsection{Integration of Graphviz with Source IMS}

We need to not only export node and arc data from Source IMS but also make the visualisation more user friendly by the exploitation of subgraphs and clusters in the visualisation of ESNs. Subgraphs play three roles in Graphviz and the implementations of visualisation of ESNs. First, a subgraph can be used to represent graph structure, indicating that certain nodes and edges should be grouped together. This is the usual role for subgraphs and typically specifies semantic information about the graph components. In the second role, a subgraph can provide a context for setting attributes. The third role for subgraphs involves the way the graph will be laid out by certain layout engines. If supported, the layout engine will do the layout so that the nodes belonging to the cluster are drawn together, with the entire drawing of the cluster contained within a bounding rectangle. The success of visualisation of ESN relies on this feature of subgraphs.

The implemented function of the output to the DOT Language, ExportToDOT, reads all the nodes and arcs and their attributes in the ESN. ExportToDOT relies on the nodes of a particular element being listed in an unbroken sequence. This allows the grouping of the nodes of each element into the subgraph clusters. The source and sink volumes that represent the inflows, storage volumes, and outflows are also attributes which GraphViz displays for each node in the ESN. The arc attributes include the starting and ending node numbers which are used by Graphviz to determine the direction of the flow. In addition, cost, capacity and flow are attributes used in arc labelling. There is a single balance node for each network used for the mass balance in an ESN. However, to make a neat and tidy presentation of ESNs, ExportToDOT creates a separate virtual balance node for each element that requires it.

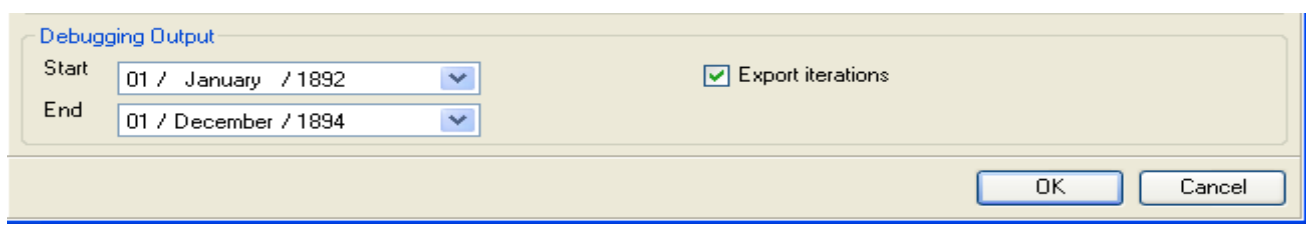

Figure 1. User interface for dumping diagnostics for ESNs

The visualisation of ESNs needs to be configured in the User Interface of Source IMS. It aims to provide users with the functionality of diagnostics of the NetLP algorithms without compromising the running performance of Source IMS. As shown in the following figure, the user interface to request the .dot files in the DOT language allows the user to turn the functionality on and off, and to configure the start and end time steps of the diagnostics. This enables a balance between water system model performance and the availability of diagnostics, because debugging users are usually only interested in a short period of time steps during the whole model simulation period. Therefore this visualisation will not cause much overhead to the running time of the software during diagnostic analysis. The created .dot files and text format files include more 
Chen et al., Visualising Equivalent System Networks in the NetLP Optimisation of Water Distribution Systems

details, such as arc numbers, and node names, dumped to a temporary directory for the visualisation of ESNs via the Graphviz tool.

Figure 2. shows an example of a simple river system built in Source IMS: (a) is the schematic view of the river system, (b) is the visualisation of the translated ESN using the exported dot file at the last iteration of the first time step. Internal nodes of a translator are clustered within a subgraph cluster. The colour of the box line of a subgraph cluster is changed from black to blue to make it easier to differentiate with arcs that are in black. The solution flows are coloured red. Users can clearly understand how the network components are translated. For example, Minimum Flow Requirement 4 is translated into two parallel arcs. One with high incentive cost and a capacity that equals to the required water 100ML, the other with zero cost and the maximum capacity to allow any excess water to pass.

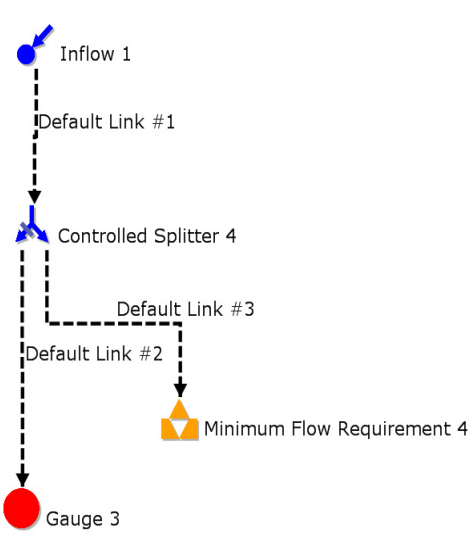

(a)

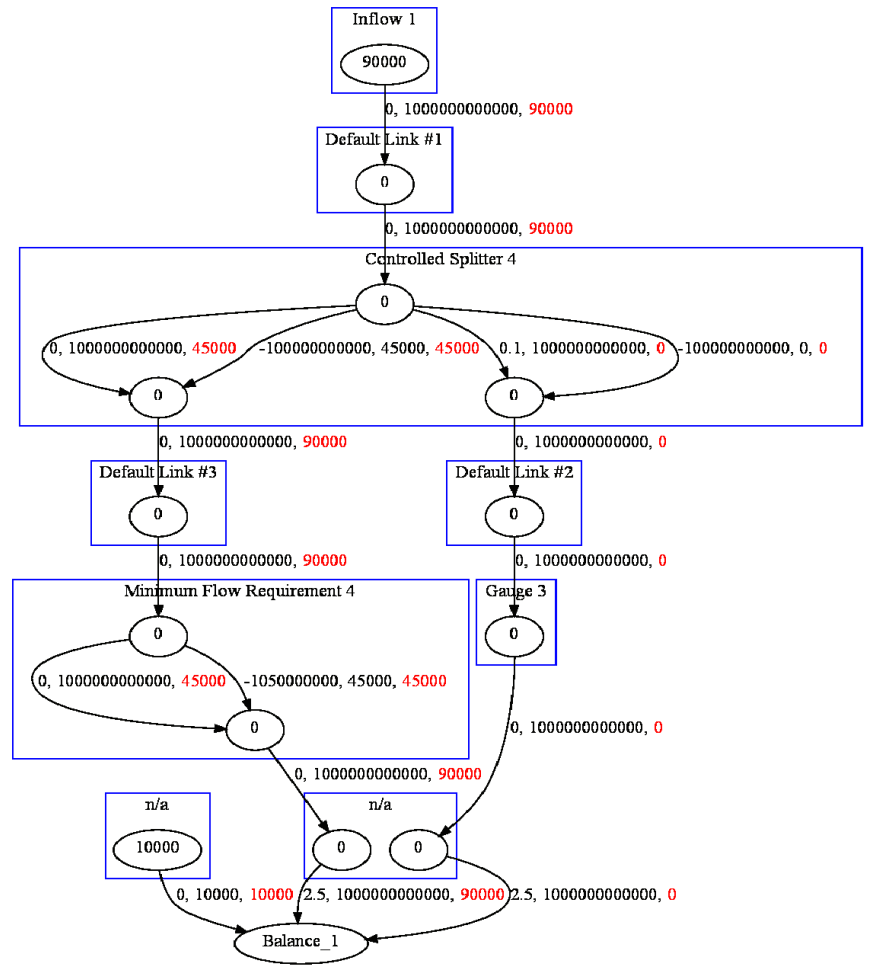

(b)

Figure 2. Example of visualisation of an ESN. Each arc is labeled with three numbers in order representing cost, capacity $\left(\mathrm{m}^{3}\right)$, and computed flow $\left(\mathrm{m}^{3} /\right.$ timestep)

\section{CASE STUDIES}

\subsection{Release Excess Water in Regulated Splitters}

The first case of the visualisation shows that a controlled splitter ESN translator needs to favour the main channel to release excess water. The regulated splitter is similar to the unregulated splitter except the user is able to define flexible flow relationships between the main and effluent flow. In Figure 3, it can be seen that the regulated splitter has cost ambiguities in distributing excess flows to the main channels, because both natural flow arcs have a cost of zero.

This would result in ambiguities in some scenarios. This situation can be avoided by adding a small cost of 0.1 to the effluent natural flow arc to ensure that excess water will by default flow down the main branch instead of the effluent. Users could still over-ride the default outcome by assigning positive costs farther down the main branch or negative costs farther down the effluent branch. Figure 4 shows a regulated splitter that is configured to have a maximum effluent flow of half the upstream flow and a minimum effluent flow of zero. The forced flow arc on the main channel (left branch) has a capacity that is the minimum flow for the main channel with a high incentive. The forced flow arc on the effluent channel (right branch) has a capacity that is equal to the minimum effluent flow, in this case zero, with a high incentive. This example shows the small cost of 0.1 on the natural flow arc on the effluent side that forces water down the main channel when the flow rules are flexible. 
Chen et al., Visualising Equivalent System Networks in the NetLP Optimisation of Water Distribution Systems

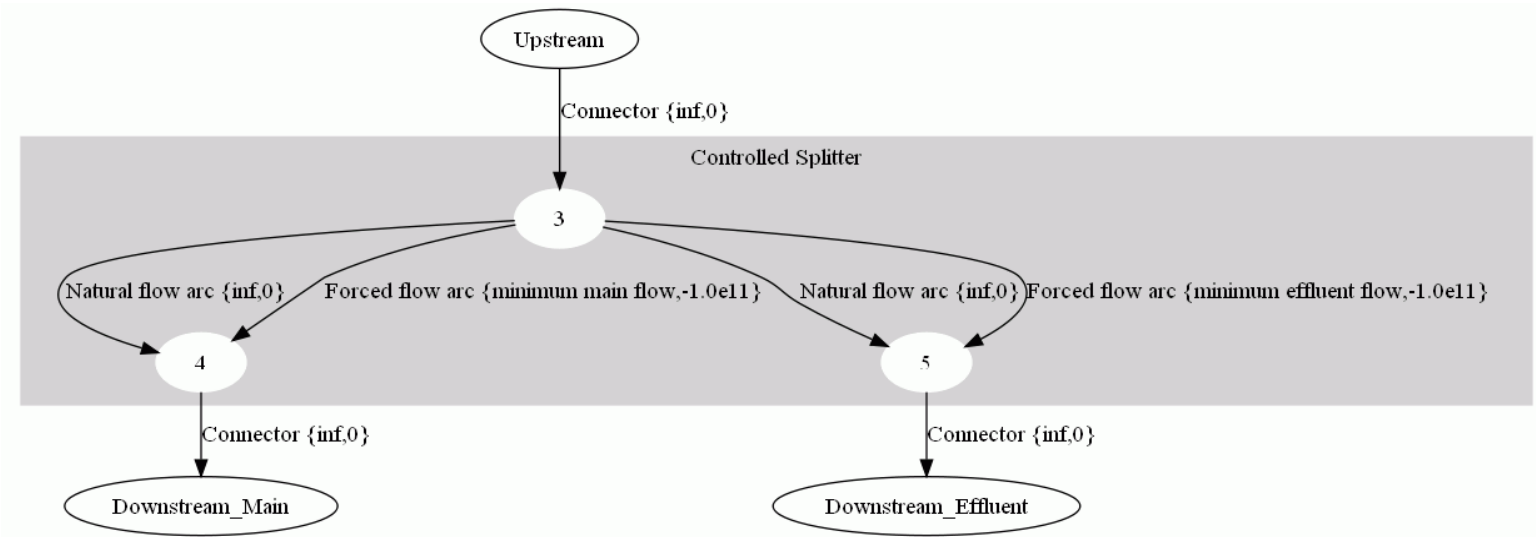

Figure 3. ESN template of Regulated Splitter node. Arc key: DESCRIPTION \{CAPACITY,COST\}, inf means the maximum capacity.

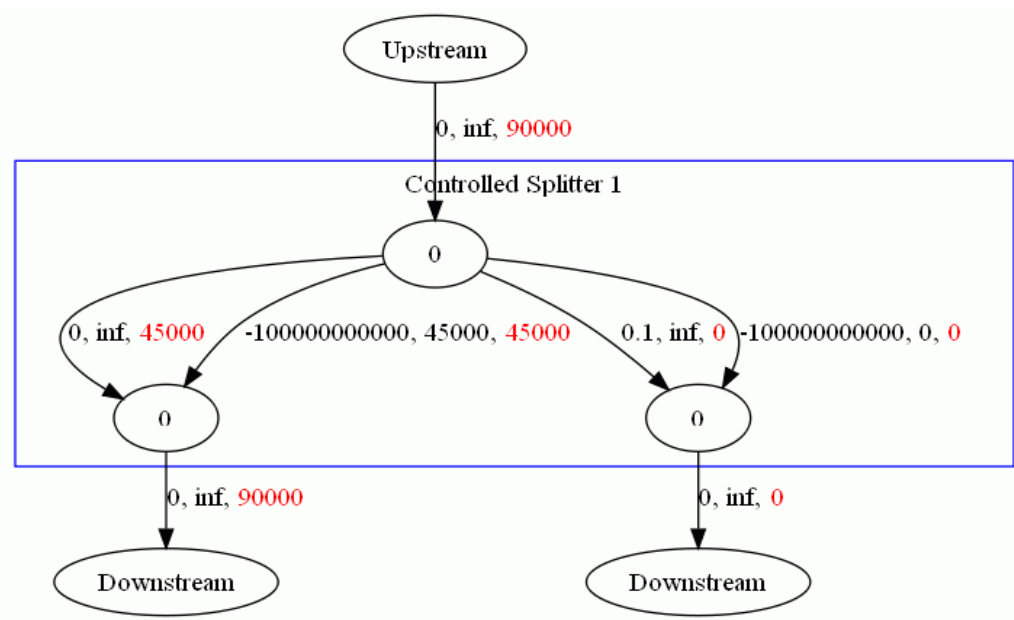

Figure 4. Regulated Splitter. Each arc is labeled with three numbers in order reperesenting NetLP cost, capacity $\left(\mathbf{m}^{3}\right)$, and computed flow $\left(\mathbf{m}^{3} /\right.$ timestep)

\subsection{Resolve Cost Ambiguities in Multiple Flow Paths}

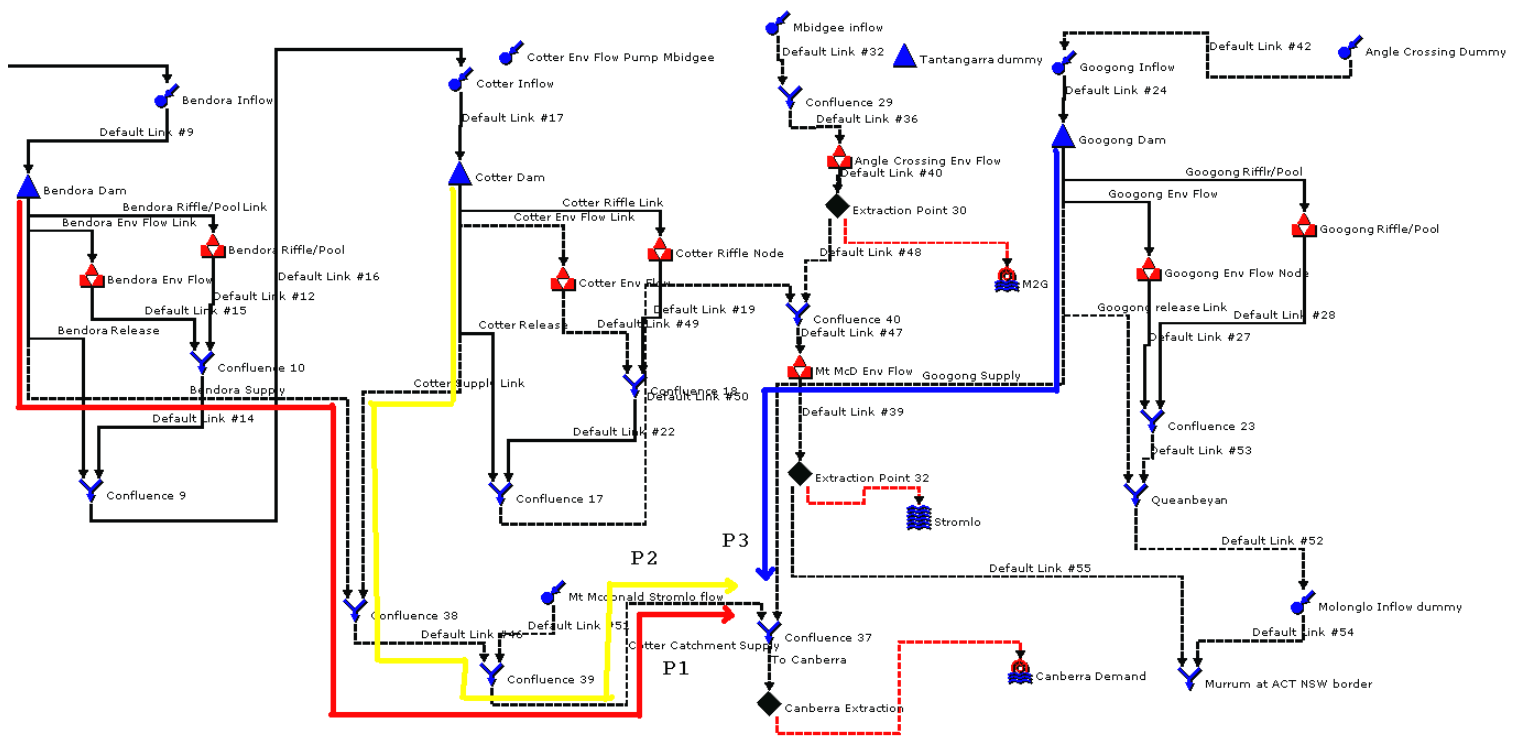

Figure 5. Multiple supply paths to Canberra Extraction 
Chen et al., Visualising Equivalent System Networks in the NetLP Optimisation of Water Distribution Systems

The second case shows how the visualisation of ESNs can help resolve cost ambiguities with multiple flow paths in Source IMS models. In Figure 5, there are three possible supply paths from three different dams to the Canberra extraction point: P1, P2, and P3. In an early version of Source IMS, it was found that at some time steps extracted water results were different with the different NetLP solvers: RELAX IV (Kuczera, 1992) and PPRN (Castro and Nabona, 1996). This issue is investigated here with the aid of a visualisation of the ESN of the translated river system.

Figure 6 shows the part of the ESN from Googong storage to Canberra Extraction, i.e. P3 path. It can be seen that all the costs along P3 - release arc of Googong, Googong Supply, Confluence 37 and Canberra Extraction, are zero. The same situations exist in P1 and P2. The cost ambiguities will allow different solutions from different NetLP solvers or running on different machines using a particular NetLP solver. For example, water can be carried over in Bendora or released to Cotter, or released to Canberra Extraction from either of them. The carryover arc (Kuczera, 1992) costs of different storages have been set up to avoid cost ambiguities, so the end storage values may always be the same, however different flow paths resulting in the same end storage values are possible.

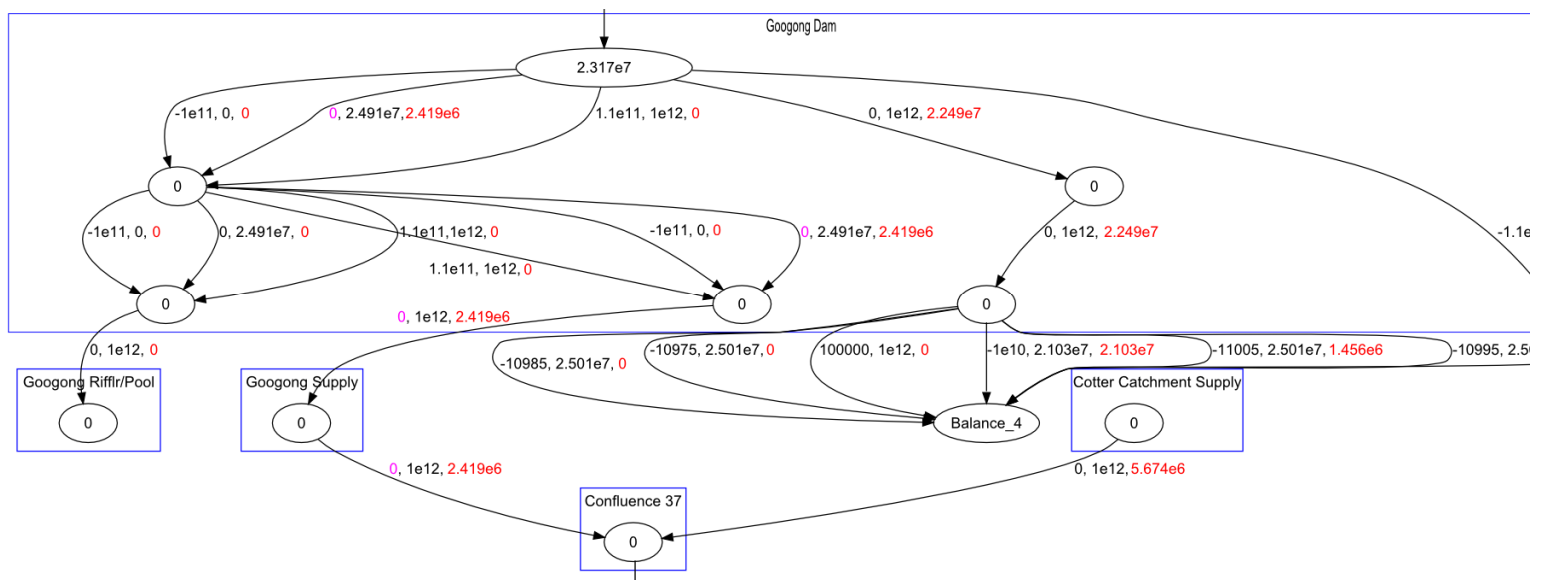

Figure 6. Visualisation of the supply path P3 in ESN. Arc key: cost, capacity $\left(\mathrm{m}^{3}\right)$, flow $\left(\mathrm{m}^{3} / \operatorname{timestep}\right)$.

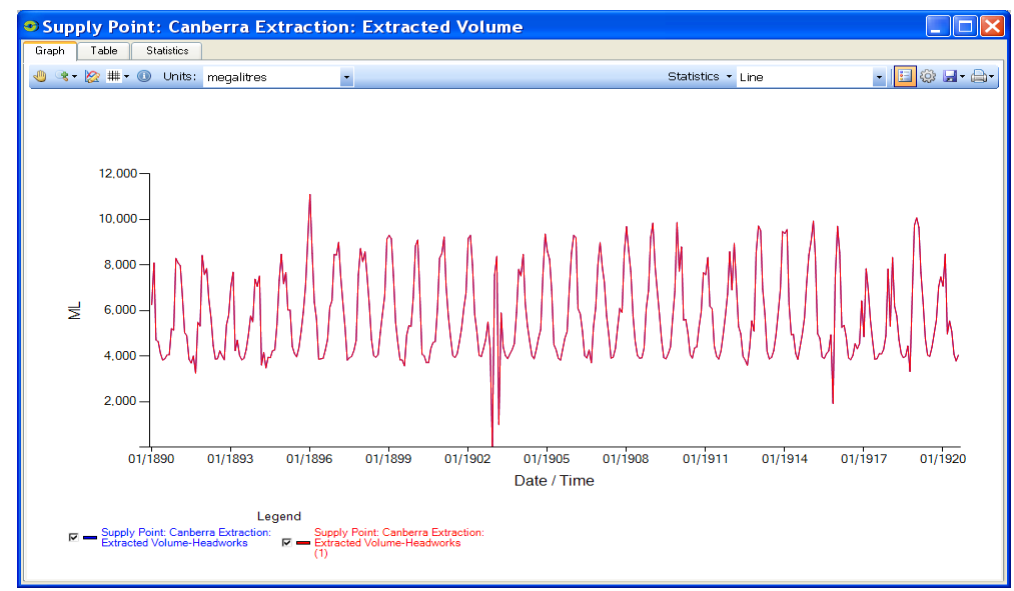

Figure 7. Consistent results using the RELAX IV and PPRN solvers.

Having identified the presence of cost ambiguities in the visualised ESN, a mitigation algorithm is implemented to successively add small incremental costs to each release arc of storages. This ensures that there are no cost ambiguities among P1, P2, and P3. As the costs are small, this won't interfere with the costs of downstream demands or carryover arcs of storages if the number of storage outlet paths is kept within a limit determined by the ratio of the minimum cost difference for demand priority and the minor cost difference in the algorithm. This implementation saves users the effort of manually adding costs to change the total costs of alternative flow paths which by default would have had cost ambiguities. Figure 7 shows that RELAX IV and PPRN algorithms get consistent results in the above project after the implementation of mitigation of cost ambiguities. 
Chen et al., Visualising Equivalent System Networks in the NetLP Optimisation of Water Distribution Systems

\section{CONCLUSIONS}

There is often a difference between the NetLP solver's solution for a network and what a water system modeller might expect. Existing software such as REALM, WathNet, MODSIM and early versions of Source IMS have no capability to visualise the generated ESNs, making it very difficult for users to understand the solutions of NetLP solvers. In this paper, visualisation of ESNs is advocated throughout the lifecycle of network LP-based modelling practices, because it can support the building, understanding and debugging of such water system models.

For this reason, Source IMS has introduced a visualisation export for the ESNs based on a third-party tool, Graphviz. This provides the modeller with an improved understanding of how the network linear optimiser is behaving in response the networks they have constructed. The usefulness of the visualisations was illustrated by two case studies using NetLP functionality in Source IMS. The first case of visualisation showed that the translation of the regulated splitter into an ESN has allowed the NetLP to make an arbitrary choice of supply path. In fact, the modeler would prefer to favour the main channel to release excess water. With this knowledge, adding a minor cost difference to the effluent arc causes Source IMS to release water down the path preferred by the modeller. The second case involved cost ambiguities in a complicated river system network with multiple storages with multiple flow paths. The visualisation showed that the multiple paths from different storages to the extraction point had exactly the same total cost. In this case, the Source IMS developers have created an automated solution to handle these cost ambiguities that works effectively in the river system network examples, thus it highlights the usefulness of the visualization of ECNs.

\section{ACKNOWLEDGMENTS}

We gratefully acknowledge the contribution of data and models from Andrew Grant (BMT WBM) and Fareed Mirza (CSIRO Land and Water). We also gratefully acknowledge the constructive comments from Geoff Adams (MDBA).

\section{REFERENCES}

Bertsekas, D. P. (1998), Network Optimization: Continuous and Discrete Models, Athena Scientific, Belmont, Mass. USA.

Castro, J. and Nabona, N. (1996). An implementation of linear and nonlinear multicommodity network flows, European Journal of Operational Research 92:37-53.

Delgado, P., Kelley, P., Murray, N. \& Satheesh, A. (2011). Source User Guide, eWater Cooperative Research Centre, Canberra, Australia

Gansner,E.R. and North,S.C. (1999). An open graph visualization system and its applications to software engineering. Softw. Pract. Exper. 00(S1), 1-5.

Graphviz. (2011) http://www.graphviz.org

Ilich, N. (2008), Shortcomings of linear programming in optimizing river basin allocation, Water Resources Research, 44

Ilich, N. (2009), Limitations of Network Flow Algorithms in River Basin Modeling, Journal of Water Resources Planning and Management. 135(48):1-8.

Jones, C. V. (1996). Visualization and optimization. Kluwer Academic Publishers, London.

Kuczera, G. (1992). Water supply headworks simulation using network linear programming. Advances in Engineering Software. 14, 55-60.

Labadie, J. W. (2004), Optimal Operation of Multireservoir Systems: State-of-the-Art Review, Journal of Water Resources Planning and Management, 130(2), 93-111.

Labadie, J. W. (2006). MODSIM: Decision Support System for Integrated River Basin Management: In Proceedings of $3^{\text {rd }}$ Biennial meeting of the International Environmental Modelling and Software Society, Vermont, USA.

Linsley, R. K., Kohler, M. A., and Paulhus, J. L. H. (1982). Hydrology for Engineers. McGraw Hill.

MODSIM. (2011). ftp://dwrftp.state.co.us/cdss/projects/MODSIM/MODSIMv8.1UserManual.pdf

Penton, D.J. and R. Gilmore. (2009). Comparing software for modelling the managementrules that river operators implement. 18th World IMACS / MODSIM Congress, Cairns, Australia, 13-17 July, 2009.

Perera, B.J.C., B. James, M.D.U. Kularathna (2005). Computer software tool REALM for sustainable water allocation and management, Journal of Environmental Management, 77(4):291-300.

REALM. (2011). http://www.water.vic.gov.au

Welsh, W, J Vaze, D Dutta, D Rassam, J Rahman, I Jolly, P Wallbrink, G Podger, M Bethune, MJ Hardy, J Teng, J Lerat (2011). An integrated modelling framework for regulated river systems, Environmental Modelling \& Software (under review). 